\title{
A Novel Multi-faceted Course Blueprint to Support Outcome-based Holistic Surgical Education: The Integrated Generation 4 Model (iG4)
}

\author{
MICHAIL SIDERIS ${ }^{1}$, VASSILIOS PAPALOIS ${ }^{2}$, THANOS ATHANASIOU ${ }^{3}$, SAVVAS PAPAGRIGORIADIS $^{4}$, \\ MICHAEL PIERIDES ${ }^{5}$, GEORGE VELMAHOS ${ }^{6}$ and APOSTOLOS PAPALOIS ${ }^{7,8}$ \\ ${ }^{1}$ Women's Health Research Unit, Queen Mary University of London, London, U.K.; \\ ${ }^{2}$ Department of Surgery and Cancer, Renal Transplant Directorate, \\ Imperial College Healthcare NHS Trust, London, U.K.; \\ ${ }^{3}$ Department of Surgery and Cancer, Cardiothoracic Directorate, \\ Imperial College Healthcare NHS Trust, London, U.K.; \\ ${ }^{4}$ International Society of Pelvic Surgery, Athens, Greece; \\ ${ }^{5}$ Kettering General Hospital NHS Foundation Trust, Kettering, U.K.; \\ ${ }^{6}$ Department of Surgery, Division of Trauma, Emergency Surgery, \\ and Surgical Critical Care, Harvard Medical School, Boston, MD, U.S.A.; \\ ${ }^{7}$ Experimental Educational and Research Centre ELPEN, Athens, Greece; \\ ${ }^{8}$ School of Medicine European University Cyprus, Nicosia, Cyprus
}

\begin{abstract}
Background/Aim: We present a novel multifaceted, internationally adaptable course curriculum blueprint, which provides holistic surgical education at the undergraduate level. Materials and Methods: The Integrated Generation 4 (iG4) course (Essential Skills in the Management of Surgical Cases - ESMSC Marathon course) curriculum consists of four essential learning components: core skills-based learning, case-based discussions, basic science workshops and soft-skills. These are all clustered in a specialty-led network architecture. Every cluster consists of modules from the four learning cores, while network nodes are modules that are mutually shared by more than one clusters. Results: We produced a standardized blueprint of 50 modules based on the 4 learning cores, covering 9 surgical specialties. This resulted in a curriculum map where every module is described using 3 parameters: $\chi$ axis (skills component), y axis (knowledge component), $z$ axis (specialty component). Conclusion: iG4 proof of concept sets the ground for a novel, reproducible and standardised effort to
\end{abstract}

This article is freely accessible online.

Correspondence to: Michail Sideris, Women's Health Research Unit, Queen Mary University of London, London, U.K. Tel: +44 7530857929,e-mail: m.sideris@qmul.ac.uk

Key Words: Surgical education, ESMSC course, surgical training, course curriculum, iG4. produce a portfolio of undergraduate surgical skills serving the vision of holistic surgical education.

Simulation-based learning (SBL) is one of the backbones of surgical education (1). SBL itself, is considered as a tool to convey knowledge and/or skills, and its application varies depending on the desirable learning outcomes. Further to this, SBL encompasses a broad-spectrum methodology targeted to cover different skills or knowledge. This can vary from simulated medical scenarios using SimMan to computerbased dry lab simulation, virtual or augmented reality simulators and cadaveric or animal-model wet lab settings. On the other hand, most surgical course curricula use a single SBL technique, they are built to run using a pre-defined infrastructure and are focused to cover a relatively narrow spectrum of learning outcomes. However, modern surgical education is shifting to achieve a more holistic model, where emphasis is given in both technical and non-technical skills as well as basic science and applied surgical knowledge (2).

Focused course curricula for undergraduates are designed to cover either a small group of technical skills or a short spectrum or non-technical skills in a fragmented manner and majorly disjoint from the required basic or applied surgical science (1). Moreover, the vast majority of these courses are built to run in a local educational facilities' setting and lack global generalizability. Notably, these efforts are focused to cover elements of a single specialty and fail to provide a helicopter view of Surgical Science in general. This restricts students from being exposed to different surgical specialties 
simultaneously, and results in a fragmented view of Surgery, which could potentially negatively influence the overall motivation towards a surgical career. And last but not least, securing funding for any educational activity especially in the context of a global economic recession, can be a massive challenge; and this explains why several Medical UK Schools have decided to shorten or even withdraw, hard-core surgical rotations in their curricula (3).

To address this, we developed a multi-faceted surgical course which aims to provide holistic surgical education for the essential surgical specialties, tailored for the undergraduates $(1,4-6)$. Our course curriculum encompasses research methodology in order to continuously evolve and support adaptability in a global setting. In this study we aim to describe in detail the blueprint of fourth generation of the course curriculum (iG4), as a global adaptable educational model for holistic surgical education.

\section{Materials and Methods}

Essential skills in the management of surgical cases - ESMSC marathon course. The course concept and curriculum evolution have been previously described in detail $(1,5,6)$. In a nutshell, ESMSC is an international bi-annual SBL course which combines small group teaching with mixed fidelity in vivo, ex vivo and dry lab simulation. The primary curriculum pillars are set to provide holistic multi-faceted surgical training in most of the surgical specialties using an internationally adaptable infrastructure. We perceive as "holistic surgical education" the provision of all the essential elements that are necessary for the modern surgeon as described by the Royal College of Surgeons (RCS) in the recent "Future of Surgery" (7); this includes technical, non-technical skills, basic science and applied clinical knowledge. Adjacent to this goal, ESMSC aims to inspire and motivate towards a surgical career and provide research opportunities through a novel research training network (8). ESMSC curriculum has undergone a continuous multifactorial evolution and its current form (Cores Integrated for Research - Ci4R) has been previously described (1). Ci4R consists of 40 modules which are split in the 4 aforementioned cores (technical, non-technical skills, basic and applied clinical science). In this manuscript, we present the forthcoming curriculum blueprint, the iG4. This is primarily built on the previous 4-learning core model, and on top of that, it encompasses a three-dimensional (3D), specialty clustered curriculum design which serves the target of "holistic education" and enhances international adaptability.

Ethics. The ESMSC application of ethical approval met directive 63/2010, PD 56/April 2013 declaration, according to local policy. The license reference number is 4857/15-09-2017, MS, AP et al.

Integrated generation 4 curriculum description. iG4 is based on 3 pillar axis x, y and z; "x axis" represents knowledge, "y axis" skills and " $z$ axis" the essential surgical specialties. Any course module can be prospectively designed using the 3 -axis pillar architecture (3D clustered architecture). This means that any module can have a unique and standardised description using the $\chi, \mathrm{y}, \mathrm{z}$ parameters as discussed thoroughly below.
" $\chi$ axis" - knowledge axis. " $\chi$ axis" can be perceived as a continuous line which includes modules that are primarily aimed to transfer knowledge; this can include basic science modules or applied clinical science or a mixture of both. The modules are classified in sub-group areas of this axis in a logical order from basic science to applied clinical science (Figure 1). We defined as "heterogeneity $\chi$ " an arbitrary parameter which reflects how heterogeneous are the relevant educational objectives (knowledge). More specifically modules that contain educational objectives that are purely basic science or applied clinical science-related, are classified as homogenous. If the educational objectives are mixed, then those modules are classified as heterogeneous. Heterogeneous modules are further classified to "mildly heterogeneous" $(<20 \%$ mixture), "moderately heterogeneous" (20-40\%) and "highly heterogeneous" (40-50\%).

The $\chi$ parameter of each module is placed on the "x axis" on an ascending heterogeneity order starting from the homogenous basic science modules and progressing to the heterogeneous modules and finally to the homogenous applied clinical science. The transition for each category is defined by a defined point on the "x axis" (Figure 1).

"y axis" - skills axis. "y axis" can be perceived as the second continuous line which includes modules that are primarily aimed to transfer skills; this can include any range from technical to nontechnical skills. The modules are classified in sub-group areas of this axis in a logical order from technical to non-technical skills. Similar to " $\chi$ axis" we defined as "heterogeneity y" an arbitrary parameter which reflects how heterogeneous are the relevant educational objectives (skills). The modules are placed on the "y axis" on an ascending order from purely homogenous technical skills modules to a mixture of technical and non-technical skills modules and finally to purely non-technical skills modules (Figure $2)$. Identical to " $\chi$ axis", heterogenous modules are further classified to "mildly heterogeneous" $(<20 \%$ mixture $)$, "moderately heterogeneous" (20-40\%) and "highly heterogeneous" (40-50\%).

"z axis" - specialty axis. "z axis" can be perceived as a third continuous line (Figure 3) which includes in an ascending order (groups z1-z6) all the surgical specialties that have been incorporated in the "ESMSC marathon course" curriculum. This was set on the assumption that we can classify our included specialties in 6 groups; the abdominal and vascular surgery (z1 group), chest surgery (z2 group), head, neck and plastics surgery (z3 group), neurosurgery (z4 group), orthopedics surgery (z5 group) and Interventional Specialties (z6 group). We defined a third arbitrary parameter, the "Affinity z" which reflects how familiar are the educational objectives of each surgical specialty; the overall clustering of these is reflected in the 6 aforementioned groups (z1-z5). "Affinity z" can have 4 values $0,1,2$ and 3. " 0 affinity" is defined when the module is specialty specific with no applicability to any other specialty (i.e. intracranial bolt insertion). "Affinity 1" is when the module can be applied to one more specialty, "affinity 2 " in 2 specialties and "affinity 3" when it is generic (i.e. suturing skills). This results in a continuous line $\mathrm{z}$ (Figure 3 ). Each module can be placed onto a group (z1- z6) if the affinity is 0 or 1 (cluster module), between groups if the affinity is 2 (intergroup modules) and in the middle of the axis if the affinity is 3 (generic modules). In certain occasions, a module can have affinity 1 with a specialty from a different group; these modules are placed in certain spaces 


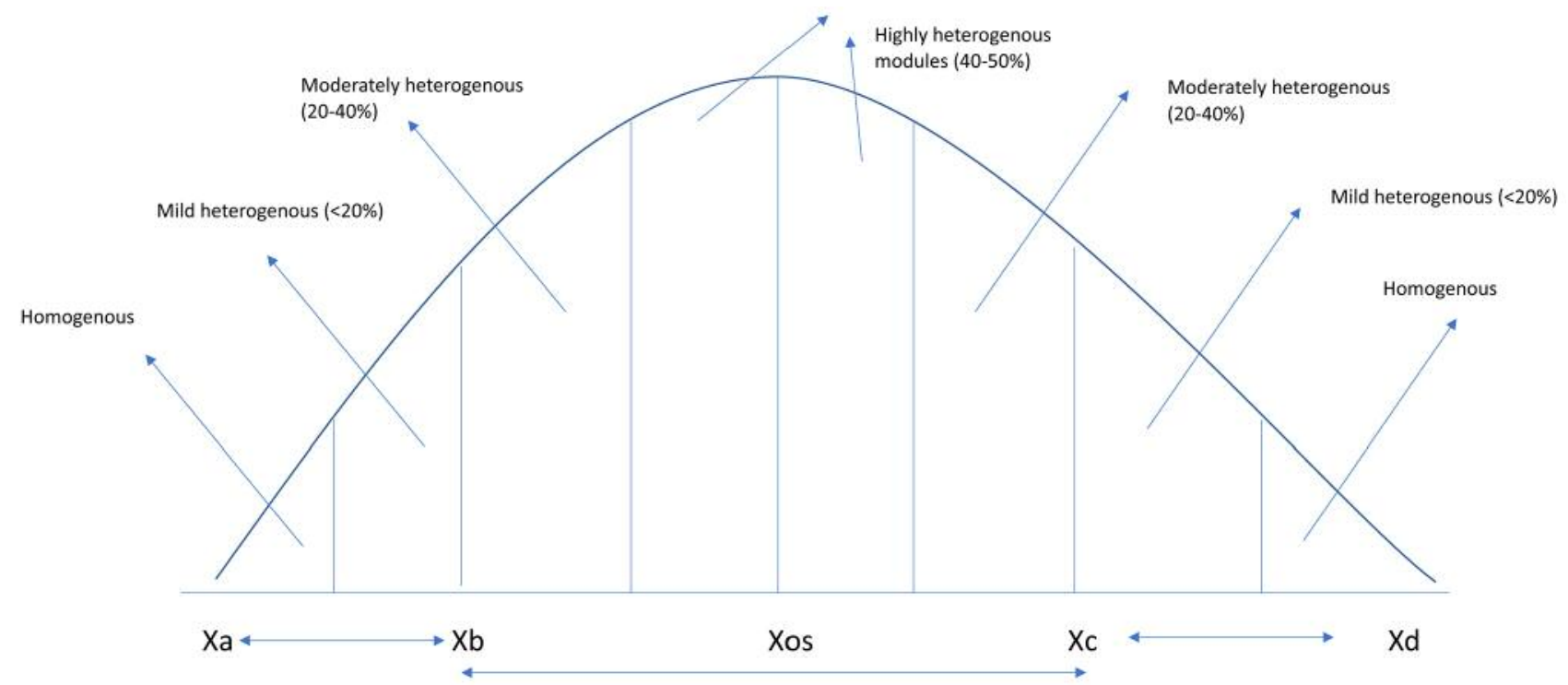

Xa-Xb: basic science modules, $\mathrm{Xc}-\mathrm{Xd}$ : applied surgical science modules, $\mathrm{Xb}-\mathrm{Xc}$ : mixed modules

$\mathrm{Xa} / \mathrm{Xd}$ : points of minimal heterogeneity in module contents, Xos: point of maximum heterogeneity

$\mathrm{Xb}$ : transition from basic science to mixed modules

Xc: transition from mixed modules to applied surgical science

We assumed heterogeneity follows a normal distribution curve

Figure $1 . \chi$ axis (knowledge).

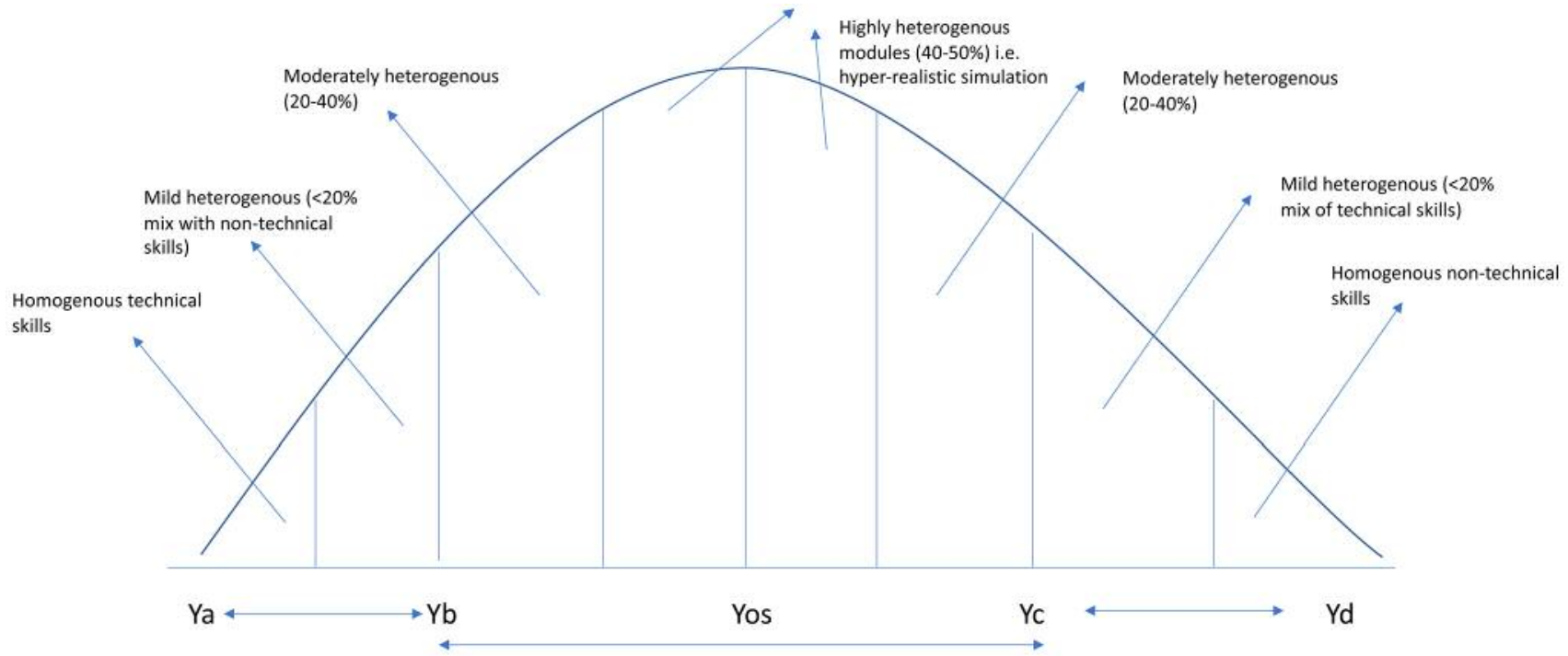

Ya-Yb: technical skills modules, $Y_{c}-Y_{d}$ : non-technical skills modules, $Y b-Y c$ : mixed skills modules

$\mathrm{Ya} / \mathrm{Yd}$ : points of minimal heterogeneity in module contents, Yos: point of maximum heterogeneity

Yb: transition from technical skills modules to mixed modules

Yc: transition from mixed modules to non-technical skills modules

We assumed heterogeneity follows a normal distribution curve

Figure 2. y axis (skills). 


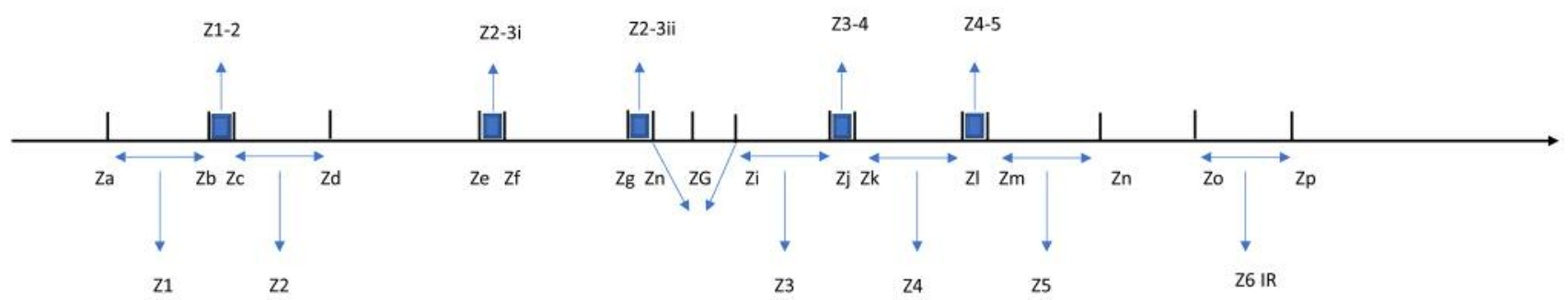

Clusters: z1, Z1-2, Z2, Z2-3i, Z2-3ii, Z3, Z3-4, Z4, Z4-5, Z5, Z6 IR

(See full text for description of each cluster)

Figure 3. $Z$ axis (Cluster axis).

of the $\mathrm{z}$ axis in between the 2 groups (z1-2, z3-4, $\mathrm{z} 4-5)$, or prior or after to "generic modules" group (z2-3i and z2-3ii) (Figure 3). "Generic modules" form an independent zG group, which is the 7th group (Figure 3). Therefore, a module can be classified in one of the possible spaces z1-z6, z1-2, z2-3i, z2-3ii, z3-4, z4-5 or zG. We did not define a z5-6 space as Interventional Specialties Skills were considered as homogenous, however, this can be modified in the future if needed. These spaces are named as "specialty clusters".

\section{Results}

Prospective module design: a step-by-step guide. The 3-axis allows a prospective systematic set up of any module in the course. Each module should include a knowledge component $(\chi$-axis) plus or minus a skills component $(y-$ axis) and be classified in one of the clusters (z-axis). The first step includes the identification of the learning objectives of each module. The second step includes the classification of the learning objectives in knowledge and/or skills. The third step goes into more depth to subclassify the learning objectives into basic science or applied surgical knowledge or mixed; equally to subclassify the applicable learning objectives into technical or non-technical skills or mixed. The fourth step is to further define the "heterogeneity $\chi$ " and the "heterogeneity $y$ " of each module. "Heterogeneity $\chi$ " is applied purely on the knowledge related learning objectives and, respectively, "heterogeneity $y$ " on the skills related learning objectives. If one of the educational components is not applicable (skills or knowledge) then the module is classified as $\mathrm{y}=0$ or $\chi=0$, respectively. Proceeding to step 5 , this includes the mapping of the educational modules in a $(\chi, y)$ 2-dimensional system where a clear helicopter view of the educational objectives is possible (Figure 4). Figure 4 indicates how a module can include any educational objective component and also, how heterogeneity reflects the direction of the module. Modules that are in the middle of the graph combine most of the possible categories of educational components; a classic example of those is the hyper-realistic simulation which requires a wide range of knowledge and skills and reflects the most accurate representation of the real clinical practice.

Following $(\chi, y)$ mapping of the module, the next step 6 is to try and categorise on the $\mathrm{z}$ axis. The process has been described in the previous section and it includes the definition of "affinity". This results in allocation of each module in one of the pre-defined clusters.

\section{Discussion}

Interpretation: the role and advantages of creating a complex $3 D$ clustered curriculum map. Prospective systematic design of the course modules results in a unique 3D identity for each of them $(\chi, y, z)$. The addition of the $z$ axis allows a harmonised cluster division of the course curriculum. This, results, in theory, in 12 clustered components of the course curriculum placed on a 3D axis system; each of them including all the essential educational objective components (knowledge, skills). The application of three possible metrics (heterogeneity $\chi, \mathrm{y}$ and affinity) allows further internal harmonization of the learning objectives in order to achieve autonomous and educationally effective 3D clusters. In simple terms, each 3D cluster can be an autonomous and purely independent curriculum. Although we have not measured the educational effectiveness of each cluster, this will be possible following prospective validation of the novel curriculum. On top of that, measuring the predefined heterogeneity and affinity metrics, this can provide an independent objective marker for the course itself.

How a 3D blueprint can serve the idea of holistic surgical education? Clearly the "ESMSC marathon course" has tried to achieve a very ambitious goal: to introduce undergraduate student in the novel concept of holistic surgical education and inspire them towards a career in any surgical specialty. Besides the running cost, which undoubtedly remains an issue in any educational effort, ESMSC had to face 2 primary challenges. The primary one is mainly described by the RCS 


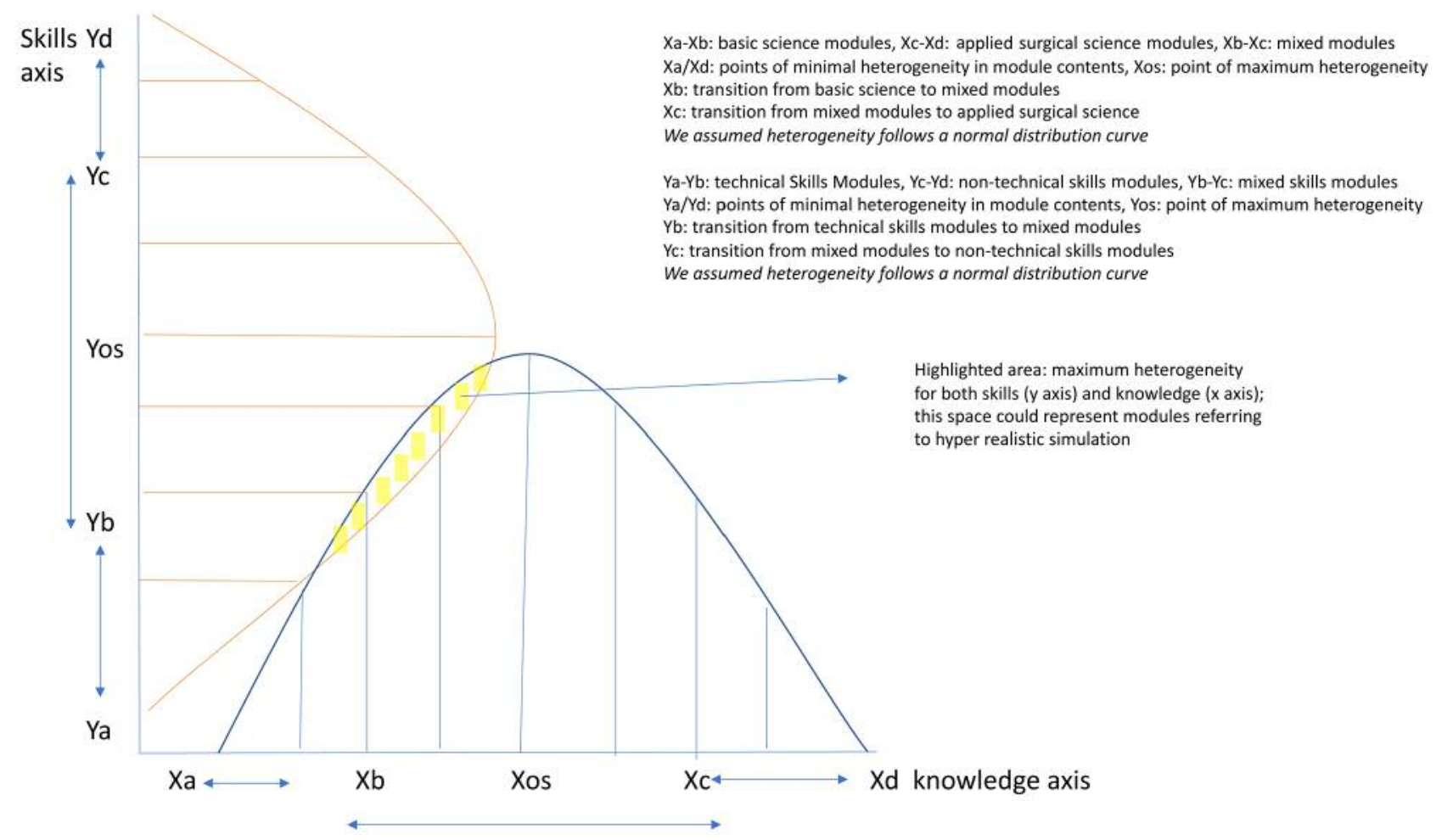

Figure 4. $(x, y)$ axis plotting representation.

in the "Future of Surgery" (7) and this includes the increasing need to accomplish the modern surgeon with a huge spectrum of skills (non-technical and technical) and knowledge (basic and applied clinical science). The second one has been the unanimous effort to include all the basic surgical specialties to maximise synchronous exposure to a wide variety of modules which could result to a helicopter view of "Surgical Science" in general. We perceived as "holistic education" those two aforementioned challenges and therefore identified the need to develop a novel curriculum architecture; this would have to be precise and internationally adaptable.

A prospective systematic methodology in modules' design based on a ( $\chi$ knowledge, y skills) and further subclassification based on two objective metrics (heterogeneity $\chi$ and y) can ultimately harmonise and maximise the spectrum of the ESMSC curriculum, and hence face our primary challenge. This has been the primitive vision of the Ci4R curriculum which is the current version of the curriculum. Further to strict metrics introduction and $(\chi, y)$ mapping which has been one of the core elements of the iG4, the introduction of a third parameter in the module design ( $\mathrm{z}$ axis) and a third metric (affinity) allowed a further systematic set up of "clusters" which reflect a specialty-led split of the course curriculum. This is promising to address the second challenge which is the incorporation of a harmonised multi-specialty curriculum.

iG4 - Can this be the basis of a global course design blueprint?. Besides facing the two aforementioned challenges, the iG4 concept has a huge potential to drive surgical courses to a novel era. Currently, most of the modern learning and teaching in Medicine follows the principles described in the relevant study of David Prideaux, who scrutinised the essential curriculum design elements (9) in order to reach a curriculum map.

Further to this, in our previous systematic review (1), we synthesised all the available "know how" to conclude to a "set up" framework guideline for undergraduate surgical courses. This incorporated 3 parameters including landscape (existing curriculum/ specialties need addressing), participation (target audience, recruitment, duration of training) and design (curriculum options, additional intervention, method of assessment, timing of assessment). The vast majority of the existing evidence to develop this framework has been low quality and lacked adaptability.

iG4 comes as a novel approach to address curriculum design. iG4 can support complex landscapes (specialties included) and curriculum maps. The introduction of 3D 
clusters can synthesise and harmonise a huge variety of SBL methods (wet, dry cadaveric simulation) and educational interventions (didactic lectures etc.) allowing complex educational goals to be achieved. In other words, iG4 can facilitate composite educational interventions allowing room for evolution and further adaptation of any novel curriculum, based on both the predefined educational metrics as well as the subjective feedback element of participants and faculty. Besides facilitating composite and wide educational efforts, iG4 can also allow "fragmentation" of big courses in small harmonised clusters which demonstrate affinity. This results in providing opportunities to conduct safely and effectively part of the ESMSC course, and not the whole syllabus, which can reduce cost and allow further adaptability to any educational setting across the world.

Ci4R versus iG4: a stepping-stone transition. Our efforts to augment and optimize simulation-based surgical education at the undergraduate level began with the introduction of 'Cores Integrated for Research' (Ci4R) curriculum, a surgical curriculum reviewed and accredited by the European Accreditation Committee for Continuous Medical Education (EACCME). CI4R forms the skeleton and overarching guide for the ESMSC Marathon course. ESMSC combines a melange of simulation modalities to achieve a unique surgical educational experience. The main driver behind the Ci4R creation was the changing surgical landscape, requiring surgical trainees with the potential to adapt and evolve at times of healthcare reforms, mounting cost-pressures and reshuffled skillsets. With 4 years of positive feedback, and outcomes mapped onto a successful progression towards a surgical career, the curriculum evolved into its latest version: the iG4. iG4's specialty clusters, as opposed to Ci4R, rely on a threedimensional educational model where different specialties form their own unique layers called clusters. The importance of this major transition from Ci4R to iG4 is manifold. Firstly, from a research perspective, this forms a unique platform upon which one can evaluate educational interventions for specific specialties to optimize knowledge and skill transfer. Secondly, this allows for the introduction of new modules in ESMSC in a systematic way that the course curriculum remains unaffected and homogenous. Lastly, iG4 allows for a more complete immersion in specific specialties such that students are fully exposed to individual surgical specialties. According to Gelder et al. (10), career choices are usually the product of three elements: longstanding interest, exposure to the job and working environment. The iG4 design, not only allows for isolated consideration of surgical specialties when it comes to choosing careers, but also helps students add a new unique dimension in their decision process: the structured and holistic 'feel' of the specialty - an element not reproducible via current medical school curricula, whilst maintain a broad appreciation of surgery and its various fields.
Limitations. We recognize a series of limitations in this concept. Firstly, this model is a theoretical description and further prospective evaluation is planned within the next 24 months. Secondly, although certain heterogeneity metrics have been applied to prospectively design any novel module, we are yet to release formal metrics for the educational effectiveness; this is going to be part of the prospective evaluation of the course, as well as part of a dedicated evidence synthesis project on assessment methodology. Lastly, iG4 application will provide with important feedback and some further minor adjustments may be applicable to meet its initial vision.

Future endeavours. The iG4 concept is built from a mathematic analogue which enhances its initial aspiration for introducing measurable metrics in composite curriculum design architecture. The short-term plan for iG4 is to code all the current modules on the $(\chi, y, z) 3 D$ axis model and collect multiple education performance markers across the time. This would allow the development of a big dataset, where application of Artificial Intelligence (AI) principles, will ultimately associate "mapped" curriculum modules with quantifiable metrics. It would also permit to measure composite inter-module interactions and therefore achieve further harmonisation of the curriculum map. The ultimate vision of iG4 is to identify and design a surgical portfolio tailored for undergraduates aimed to support holistic surgical education.

\section{Conflicts of Interest}

The Authors have no conflicts of interest to declare regarding this study.

\section{Authors' Contributions}

Michail Sideris (MS) has conceived and developed the Integrated Generation 4 (iG4) course curriculum. MS and Professor Apostolos Papalois (AP) are founders and currently leading the Essential Skills in the Management of Surgical Cases - ESMSC Marathon Course (esmsc.gr). MS has drafted the manuscript; Professor Vassilios Papalois, Mr Savvas Papagrigoriadis, Dr Michael Pierides, Professor Thanos Athanasiou and Professor George Velmahos are senior advisors of the ESMSC curriculum with major contribution in the evolution and final delivery of the iG4 model. All Authors have edited and approved the final manuscript.

\section{References}

1 Theodoulou I, Nicolaides M, Athanasiou T, Papalois A and Sideris M: Simulation-based learning strategies to teach undergraduate students basic surgical skills: A systematic review. J Surg Educ 75(5): 1374-1388, 2018. PMID: 29422405. DOI: 10.1016/j.jsurg.2018.01.013

2 Sadideen H, Alvand A, Saadeddin M and Kneebone R: Surgical experts: Born or made? Int J Surg 11(9): 773-778, 2013. PMID: 23838344. DOI: 10.1016/j.ijsu.2013.07.001 
3 London KsC: Curriculum 2020 reformation (mbbs). King's College London, 2015. Available at: https://www.kcl.ac.uk/lsm/research/ divisions/hscr/study/undergradops/kumec/mbbs-2020

4 Sideris M, Papalois A, Theodoraki K, Dimitropoulos I, Johnson EO, Georgopoulou EM, Staikoglou N, Paparoidamis G, Pantelidis P, Tsagkaraki I, Karamaroudis S, Potoupnis ME, Tsiridis E, Dedeilias P, Papagrigoriadis S, Papalois V, Zografos G, Triantafyllou A and Tsoulfas G: Promoting undergraduate surgical education: Current evidence and students' views on esmsc international wet lab course. J Invest Surg 30(2): 71-77, 2017. PMID: 27611894. DOI: 10.1080/08941939.2016.1220652

5 Sideris M, Papalois A, Tsoulfas G, Majumder S, Toutouzas K, Koletsis E, Dedeilias P, Lymperopoulos N, Papagrigoriadis S, Papalois V and Zografos G: Developing an international combined applied surgical science and wet lab simulation course as an undergraduate teaching model. Biomed Res Int 2015: 463987, 2015. PMID: 26613083. DOI: 10.1155/2015/463987

6 Sideris M, Hanrahan J, Tsoulfas G, Theodoulou I, Dhaif F, Papalois V, Papagrigoriadis S, Velmahos G, Turner P and Papalois A: Developing a novel international undergraduate surgical masterclass during a financial crisis: Our 4-year experience. Postgrad Med J 94(1111): 263-269, 2018. PMID: 29519810. DOI: 10.1136/postgradmedj-2017-135479
7 Royal College of Surgeons (RCS): Future of surgery. RCS Bulletin Special Issue, 2019. Available at: https://futureo fsurgery.rcseng.ac.uk

8 Sideris M, Hanrahan J, Staikoglou N, Pantelidis P, Pidgeon C, Psychalakis N, Andersen N, Pittaras T, Athanasiou T, Tsoulfas $\mathrm{G}$ and Papalois A: Optimizing engagement of undergraduate students in medical education research: The emerg training network. Ann Med Surg (Lond) 31: 6-10, 2018. PMID: 29922460. DOI: 10.1016/j.amsu.2018.05.008

9 Prideaux $\mathrm{D}$ : $\mathrm{Abc}$ of learning and teaching in medicine. Curriculum design. BMJ 326(7383): 268-270, 2003. PMID: 12560283. DOI: $10.1136 / \mathrm{bmj} .326 .7383 .268$

10 Gelder C, Selwyn-Gotha J, Rouhani M and Rufai S: Which factors influence career choice? A medical student survey. Int J Surg 36: S103-S104, 2016. DOI: 10.1016/j.ijsu.2016.08.365

Received November 29, 2019

Revised December 4, 2019

Accepted December 6, 2019 